\title{
Dengue-specific subviral nanoparticles: design, creation and characterization
}

\author{
Niyati Khetarpal ${ }^{1 \dagger}$, Ankur Poddar ${ }^{1 \dagger}$, Satish K Nemani ${ }^{1,2+}$, Nisha Dhar ${ }^{1}$, Aravind Patil ${ }^{1}$, Priyanka Negi ${ }^{1}$, \\ Ashiya Perween', Ramaswamy Viswanathan', Heinrich Lünsdorf ${ }^{3}$, Poornima Tyagi', Rajendra Raut', Upasana Arora', \\ Swatantra K Jain ${ }^{4}$, Ursula Rinas ${ }^{2,3}$, Sathyamangalam Swaminathan ${ }^{1 *}$ and Navin Khanna ${ }^{1 *}$
}

\begin{abstract}
Background: Dengue is today the most significant of arboviral diseases. Novel tools are necessary to effectively address the problem of dengue. Virus-like particles (VLP) offer a versatile nanoscale platform for developing tools with potential biomedical applications. From the perspective of a potentially useful dengue-specific tool, the dengue virus envelope protein domain III (EDIII), endowed with serotype-specificity, host receptor recognition and the capacity to elicit virus-neutralizing antibodies, is an attractive candidate.

Methods: We have developed a strategy to co-express and co-purify Hepatitis B virus surface (S) antigen in two forms: independently and as a fusion with EDIII. We characterized these physically and functionally.

Results: The two forms of the $\mathrm{S}$ antigen associate into VLPs. The ability of these to display EDIII in a functionally accessible manner is dependent upon the relative levels of the two forms of the $S$ antigen. Mosaic VLPS containing the fused and un-fused components in 1:4 ratio displayed maximal functional competence.
\end{abstract}

Conclusions: VLPs armed with EDIII may be potentially useful in diagnostic, therapeutic and prophylactic applications.

Keywords: Dengue envelope domain III, Hepatitis B surface antigen, Virus-like particle, Bionanoparticles, Pichia pastoris

\section{Background}

Currently, dengue represents the most important arboviral disease that places nearly half the global population at risk [1]. The mosquito-borne disease is caused by four closely related, yet antigenically distinct, serotypes of dengue viruses (DENV-1, -2, -3 and -4) [2]. All four DENVs and their mosquito vectors are co-prevalent in more than one hundred tropical/sub-tropical countries. Each of the DENVs can cause disease ranging from mild dengue fever to severe dengue hemorrhagic fever and potentially fatal dengue shock syndrome [3]. Tools for diagnosing, treating and ultimately preventing dengue are urgently needed [4]. While increasingly reliable diagnostic tools are becoming available [4-6], antivirals $[4,7,8]$ and vaccines $[9,10]$ for dengue continue to be elusive.

\footnotetext{
* Correspondence: swami@icgeb.res.in; navin@icgeb.res.in

${ }^{\dagger}$ Equal contributors

${ }^{1}$ Recombinant Gene Products Group, International Centre for Genetic Engineering \& Biotechnology, Aruna Asaf Ali Marg, New Delhi 110067, India Full list of author information is available at the end of the article
}

Nanobiotechnology, which seeks to use naturally occurring as well as engineered nanoscale biomaterials to make functional systems, is rapidly emerging as a platform for the development of novel nanotools with potential biomedical applications [11-13]. We are interested in exploring potential applications of this technology to infectious diseases. We have utilized $\mathrm{Eu}^{3+}$-doped polystyrene nanoparticles as very sensitive reporters for detecting Hepatitis B virus [14] and human immunodeficiency virus [15] infections. We have also used nanoparticles of biological origin, namely, virus-like particles (VLPs). Many viral capsid proteins possess intrinsic ability to self-assemble into VLPs when expressed in recombinant insect, yeast and mammalian host systems [13]. Recently, we have begun exploring VLP platforms for the display of foreign antigens [16]. The foreign antigen we focus on is derived from the major DENV structural antigen on the virion surface, the envelope protein. Multiple antigenic determinants that are largely serotype-specific map to a C-terminal $\sim 100$ amino acid (aa) region of this protein 
[17]. Further these antigenic determinants tend to elicit DENV-neutralizing antibodies and coincidentally, this C-terminal region, which is known as envelope domain III (EDIII), is also implicated in host receptor recognition [18]. For these reasons, we believe EDIII offers an attractive precursor for developing nanoparticle tools that may be useful in addressing the problem of dengue.

Recombinant Hepatitis B virus surface (S) antigen is well-documented to self-assemble into 20-22 nm VLPs [19-21] and is the main component of commercial Hepatitis B vaccines [22,23]. In this study, we sought to exploit the $\mathrm{S}$ antigen VLP as a carrier for DENV-2 EDIII. To this end we fused EDIII to the amino-terminus of $\mathrm{S}$ to create a fusion antigen, herein referred to as ES antigen. We used the methylotrophic yeast Pichia pastoris, which we have found earlier to express $\mathrm{S}$ antigen VLPs to very high levels [20], to express the ES antigen. We found that for DENV-2 EDIII to be displayed on the VLP surface in way that made it accessible to DENV-2-specific antibody and to the host cell receptor, it was necessary to co-express the ES fusion antigen with un-fused $S$ antigen. We describe our strategy to co-express ES and $\mathrm{S}$ antigens in $P$. pastoris, their co-purification and structural as well as functional characterization of the resultant mosaic ES,S VLPs.

\section{Results}

\section{Strategy to co-express ES fusion antigen gene in the background of 0-4 copies of $S$ gene}

The ES fusion antigen was designed to contain the DENV-2 EDIII (spanning aa residues 297-400 of the full-length envelope protein), linked in-frame to the aminoterminus of the Hepatitis B S antigen through a pentapeptide linker (Additional file 1: Figure S1). The ES gene was placed under the transcriptional control of the $P$. pastoris alcohol oxidase $1(A O X 1)$ promoter. This construct is designed to express a $\sim 37 \mathrm{kDa}$ ES antigen with the first 104 and the last 226 aa residues representing, respectively, the DENV-2 EDIII and S fusion partners, in the absence of any un-fused $S$ antigen. As the ES antigen by itself did not form VLPs efficiently (see below), we attempted to co-express varying levels of un-fused $S$ antigen as well. For this, 1, 2 and 4 copies of an $S$ gene expression cassette (once again, $A O X 1$ promoter-driven), were inserted at the 5' end of the ES expression cassette in a sequential head-to-tail manner, using an in vitro multimerization strategy described previously [19]. The $\mathrm{S}$ antigen expression cassette is designed to generate a $\sim 24 \mathrm{kDa}$ protein, whose aa sequence is identical to the C-terminal 226 aa residues of the ES antigen. A schematic representation of the basic map of these constructs is depicted in Figure 1A. Putative VLPs that could arise out of ES antigen in the absence of any $\mathrm{S}$ antigen co-expression (designated $\mathrm{ES}, \mathrm{S}_{0}$ ), and in the presence of 1,2 and 4 copies of the $S$ antigen, designated as $E S, S_{1}, E S, S_{2}$ and $E S, S_{4}$, respectively, are shown schematically in Figure $1 \mathrm{~B}$.

\section{Creation of $P$. pastoris clones designed to co-express ES, $\mathrm{S}_{0-4}$ antigens}

The ES gene fusion constructs in the background of 0-4 copies of $S$ gene, described above, were integrated into $P$. pastoris using standard methods. Selected transformants representing each of the constructs were analysed for ES and $S$ antigen expression upon methanol induction. The results of a typical experiment analysing the polypeptide profiles of induced cell lysates are shown in Figure 2A. Upon induction, a new polypeptide band of $\sim 24 \mathrm{kDa}$, consistent with the predicted size of the $\mathrm{S}$ antigen, is apparent in all clones harboring the $S$ gene (compare lanes '1-4' with lane ' $U$ '). As expected, the clone lacking the $S$ gene did not express the $24 \mathrm{kDa}$ band (compare lanes ' $U$ ' with ' 0 '). Interestingly, the band intensity of this $\sim 24 \mathrm{kDa}$ polypeptide seemed to reflect the $S$ gene copy number, being indiscernible in the $S$ gene-lacking clone (lane ' 0 '), but maximal in the clone harbouring 4 copies of the $S$ gene (lane ' 4 '). However, it was difficult to unambiguously detect the presence of a $\sim 37 \mathrm{kDa}$ protein band, the predicted size of the ES antigen, in these clones. To confirm if indeed the ES antigen is expressed by these clones we carried out an immunoblot assay using an in-house $S$ antigen-specific mAb 5S, which is expected to recognize both ES as well as S antigen polypeptides. The data unambiguously revealed the presence of $\sim 37 \mathrm{kDa} E S$ antigen in the induced lysates of all four clones (Figure 2B). As expected three of these clones also co-expressed the $\sim 24 \mathrm{kDa} \mathrm{S}$ antigen. Interestingly, once again, the levels of $S$ antigen manifested a clearly visible copy number effect. A densitometric analysis of the relative intensities of the $\sim 24 \mathrm{kDa}$ bands in the 1,2 and 4 copy clones (Figure 2B, lanes '1,' 2 ' and ' 4 ') was found to be $1,1.8$, and 3.9 , respectively, closely matching the corresponding $S$ gene copy number for these three clones. This copy number effect is consistent with our earlier observation which showed that increasing $S$ gene copy number is paralleled by a corresponding increase in $S$ mRNA and protein levels [19]. Further, a densitometric comparison of ES and $\mathrm{S}$ antigen expression levels also showed that the S/ES ratios were $0.8,2.4$ and 3.7, respectively, for $E S, S_{1}, E S, S_{2}$ and $E S, S_{4}$, This is in good agreement with the predicted ratios of 1,2 and 4 , for ES, $S_{1}, E S, S_{2}$ and $\mathrm{ES}_{3} \mathrm{~S}_{4}$, respectively. Performing the immunoblot using an in-house EDIII-specific mAb 24A12 recognized only the $\sim 37 \mathrm{kDa}$ band as expected (Figure $2 \mathrm{C}$ ). The observed immunoreactivities of the $\sim 37$ and $\sim 24 \mathrm{kDa}$ polypeptides are consistent with those of ES and $\mathrm{S}$ antigens, respectively. Collectively, these data confirmed that our panel of $P$. pastoris clones co-express ES and S antigen, the latter at calibrated levels through pre-determined gene copy 

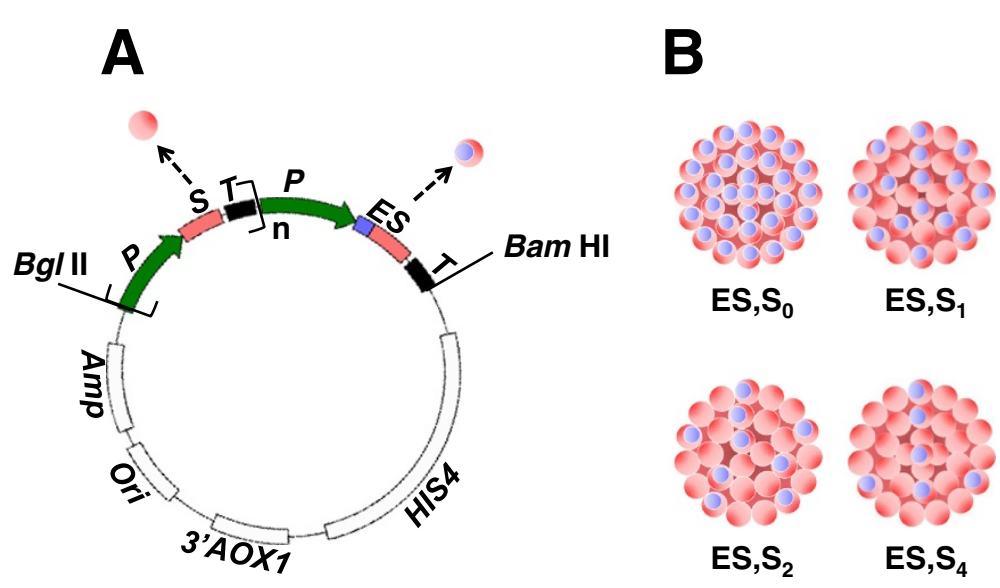

Figure 1 Strategy to produce DENV-2 EDIII containing VLPs in P. pastoris. (A) Map of pAO815-based P. pastoris-integrative expression vector carrying HBsAg $(S)$ and EDIII-2-HBsAg (ES) antigen gene expression cassettes in tandem. Each gene is present in an independent expression cassette with the AOX1 promoter $(P)$ on the $5^{\prime}$ side and the AOX1 transcription terminator ( $T$ ) on the $3^{\prime}$ side. A family of vectors was created in which the number $(n)$ of $S$ gene expression cassettes varied from $0-4$. Other elements present in the vector include the ampicillin resistance marker (Amp), the bacterial replication origin (Ori), $3^{\prime}$ terminal sequences of the AOX1 gene (3'AOX 1$)$ and the yeast histidinol dehydrogenase marker gene (HIS4). The dashed arrows point to symbols used to depict the proteins encoded by the ' $S$ ' (red sphere) and 'ES' (red and blue sphere) genes. Unique sites used for cassette assembly are shown. (B) Schematic representations of the putative VLPs predicted to be generated from the construct when the ' $S$ ' antigen copy number is $0\left(E S, S_{0}\right), 1\left(E S, S_{1}\right), 2\left(E S, S_{2}\right)$ or $4\left(E S, S_{4}\right)$ per copy of the 'ES' fusion antigen.

number establishment. It is to be noted that the constructs and $P$. pastoris clones described in this manuscript are based on DENV-2 EDIII. We have extended this approach to the EDIIIs of the remaining three DENV serotypes as well and generated corresponding $P$. pastoris clones (data not shown).

The ES, $\mathrm{S}_{0-4}$ antigens are predominantly associated with the $P$ fraction of induced cell lysates

It has been reported that the $S$ antigen expressed in yeast associates closely with the cellular membrane components [21,24,25]. This would predict that the ES antigen may also manifest this tendency. To investigate this, we analysed the distribution of the ES antigen between the soluble and membrane-enriched $\mathrm{P}$ fractions of induced cell lysates. A typical localization experiment is shown in Figure 3A. Induced total cell lysates (T) were separated into supernatant $(\mathrm{S})$ and pellet $(\mathrm{P})$ fractions, and all three samples of each induced clone were evaluated by immunoblot assay using mAb 24A12. This revealed that regardless of $\mathrm{S}$ antigen co-expression, the ES antigen was almost exclusively associated with the P fraction. We next

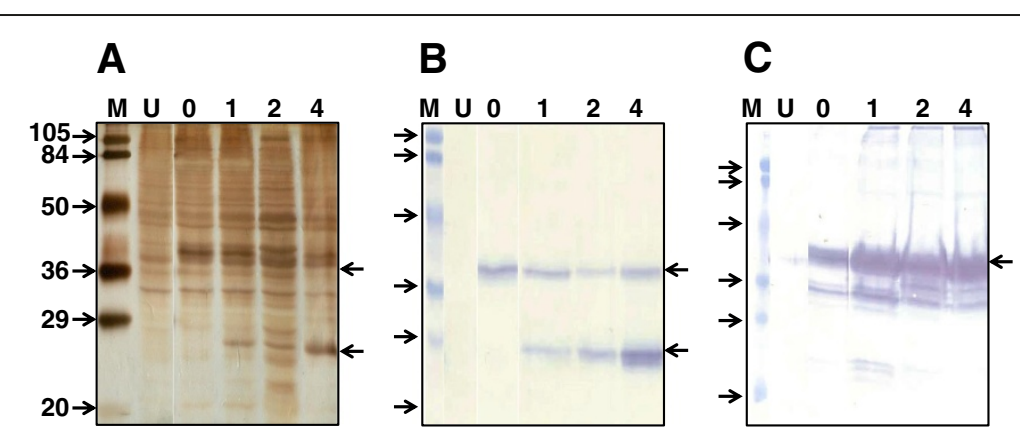

Figure 2 Co-expression of ES fusion antigen with $\mathbf{0 - 4}$ copies of $\mathbf{S}$ antigen in $\mathbf{P}$. pastoris. (A) Methanol-induced $P$. pastoris clones harboring the ES fusion gene in the background of $0,1,2$ and 4 copies of the $S$ gene were lysed and run on SDS-PAGE (lanes are indicated by the $S$ gene copy number), followed by silver staining. An un-induced sample (of clone $E S, S_{4}$ ) was analysed in lane ' $U$ '. (B) The same samples as in ' $A$ ' were transferred onto a nitrocellulose membrane after SDS-PAGE, and subjected to immunoblotting with mAb 5S. (C) Immunoblot analysis similar to that shown in panel ' $\mathrm{B}$ ', but probed using mAb 24A12. In all three panels, pre-stained protein markers were run in lanes ' $\mathrm{M}$ '; their sizes in $\mathrm{kDa}$ are shown to the left of panel ' $A$ '. The arrows to the right indicate the positions of the ES fusion (upper) and the $S$ (lower) antigens. 


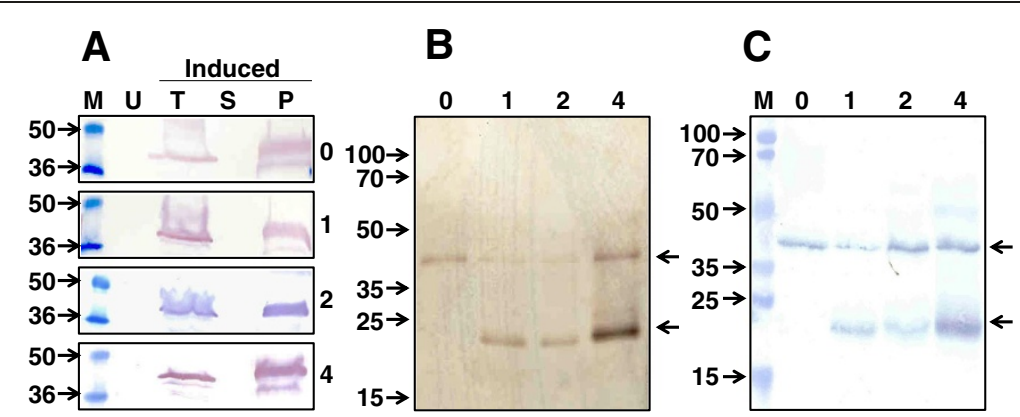

Figure 3 Purification of the $P$. pastoris-expressed $\mathrm{ES}, \mathrm{S}_{0}, \mathrm{ES}, \mathrm{S}_{1}, \mathrm{ES}, \mathrm{S}_{2}$, and $\mathrm{ES}, \mathrm{S}_{4}$, antigens. (A) Localization of the recombinant $\mathrm{ES}$ fusion antigens by Western blot analysis using mAb 24A12. Four ES fusion protein-expressing P. pastoris clones, co-expressing 0, 1, 2 and 4 copies of the $S$ antigen (indicated by Arabic numerals on the right side of the immunoblot strips) were analysed. Aliquots of $P$. pastoris cultures were analysed before $(U)$ and after methanol induction; induced cell extracts were analysed before $(T)$ and after separation into supernatant $(S)$ and pellet $(P)$ fractions. Pre-stained protein markers were analysed in lanes ' $M$ '. Their sizes (in $\mathrm{kDa}$ ) are indicated to the left. (B) Polypeptide profiles of the recombinant ES, $S_{0}$ (lane ' $0^{\prime}$ ), ES, $S_{1}$ (lane ' 1 '), ES, $S_{2}$ (lane ' 2 '), and ES, $S_{4}$ (lane ' 4 '), antigens purified from the ' $P^{\prime}$ fractions of the induced cells (described in panel ' $\mathbf{A}$ '), visualized by silver staining. (C) Western blot analysis of the purified proteins shown in panel 'B'', using mAb 5S. Pre-stained protein markers were run in lane ' $\mathbf{M}$ '. All other lanes are similar to panel ' $\mathbf{B}$ '. For panels ' $\mathbf{B}$ ' and ' $\mathbf{C}$ ', the arrows on the left indicate the positions and sizes (in $\mathrm{kDa}$ ) of the protein markers analysed in parallel; the arrows to the right indicate the positions of the ES (upper) and $\mathbf{S}$ (lower) antigens.

sought to quantify the levels of the recombinant antigens in the P fraction using a sandwich ELISA approach. Antigens were captured using either EDIII-specific or S-specific $\mathrm{mAb}$ and revealed using a second $\mathrm{S}$ antigen-specific $\mathrm{mAb}$ conjugate (Additional file 1: Figure S2). This experiment confirmed the association of both ES and $\mathrm{S}$ antigens almost exclusively with the $\mathrm{P}$ fraction for all clones. In line with expectation, when recombinant antigens were captured using $\mathrm{mAb}$ specific to $\mathrm{S}$ antigen, we observed a copy number-dependent increase in antigen levels. However, it is to be noted that this ELISA recognizes both ES and $\mathrm{S}$ antigens. Interestingly, even though all $P$. pastoris clones were designed to express ES antigen from a single copy gene, we found ES levels paralleled the increase in $\mathrm{S}$ antigen gene copy number, when mAb $24 \mathrm{~A} 12$ was used for capture. We interpret this data to reflect more efficient capture of ES antigen in the sandwich ELISA as the levels of co-expressed $\mathrm{S}$ antigen increases.

\section{Co-purification of the ES and $\mathrm{S}$ antigens from induced $P$. pastoris cells}

As a prelude to purification, we sought to optimize the conditions of induction. To this end, methanol concentration and induction duration were varied with respect to each other, followed by analysis of ES antigen in the P fractions by immunoblotting and ELISA. Based on these results (Additional file 1: Figure S3), we carried out induction at $1 \%$ methanol for 3 days. As the ES antigen was predominantly associated with the $\mathrm{P}$ fraction, we decided to adapt a protocol recently developed in our laboratory that exploits a similar behaviour of the $\mathrm{S}$ antigen for its purification [21]. Thus, the $\mathrm{P}$ fraction from induced cells which served as the starting material was solubilized in the presence of detergent and urea, subjected to polyethylene glycol 6000 (PEG 6000) precipitation, tangential flow filtration (TFF) using a $300 \mathrm{kDa}$ cutoff membrane, and finally chromatography on Phenyl Sepharose. The peak fractions were pooled and aliquots analysed by silver stained SDS-PAGE as shown in Figure 3B. The data reveal that both the ES and $S$ antigens expressed by each of the ES, $\mathrm{S}_{1-4}$ clones copurified through the multistep purification protocol. A densitometric scan revealed $>95 \%$ purity for each. That the co-purified proteins were indeed ES and S was confirmed by their identification in a Western blot analysis using mAbs 5S (Figure 3C) and 24A12 (data not shown). Importantly, both the silver stained gel and the immunoblot analyses revealed the copy number effect in $\mathrm{S}$ antigen levels. To explore if purification could be achieved in the absence of urea, we used an alternate protocol in which the starting material was the soluble fraction obtained from total cell lysate prepared in the presence of $0.6 \%$ Tween 20 [25]. This resulted in low yields (data not shown), commensurate with the low concentrations in the $\mathrm{S}$ fractions (Additional file 1: Figure S2).

\section{Co-expressed ES and S antigens form VLPs}

The demonstration that ES and S antigens could be copurified, particularly in a protocol that involved TFF across a $300 \mathrm{kDa}$ cut-off membrane suggested that the ES and S antigens are closely associated with each other and presumably incorporated into higher order structures. To investigate this further, we analysed a partially purified preparation (obtained after the TFF step) from the ES, $\mathrm{S}_{4}$ clone on a $\mathrm{CsCl}$ density gradient. Fractions were analysed in ES- and S-specific sandwich ELISAs, referred to above. This revealed that both ES and $\mathrm{S}$ antigens co-sedimented 
together in the bottom one-third of the gradient. An aliquot of this fraction containing both ES and S peaks by electron microscopy revealed the presence of discrete VLPs (Additional file 1: Figure S4). To answer the question if VLP formation occurs when the levels of S antigen co-expression was lower, aliquots of the pooled peak phenyl sepharose fractions from each of the four preps was analysed by electron microscopy (EM) as shown in Figure 4 . This study revealed that the ES, $\mathrm{S}_{0}$ antigen preparation appeared to contain large polydisperse aggregates without any discrete VLPs. Given that the $S$ antigen expressed in P. pastoris forms VLPs efficiently [20,21], this observation may likely indicate that the EDIII fusion partner in the ES antigen hinders efficient VLP formation. However, the ability to form VLPs was visibly enhanced with increasing levels of co-expressed $\mathrm{S}$ antigen. Thus, the VLPs in the ES, $\mathrm{S}_{4}$ prep manifested the presence of well-formed VLPs with the highest degree of homogeneity. Taken together, the purification and EM data show that the ES, $\mathrm{S}_{0}$ represents aggregates containing only the ES fusion antigen, whereas the ES, $\mathrm{S}_{1}, E S, \mathrm{~S}_{2}$ and $E S, S_{4}$ represent mosaic VLPs containing progressively increasing proportions of $S$ antigens as well. Further, the incorporation of ES into VLP is progressively improved by increasing the relative proportion of $\mathrm{S}$ antigen. Similar VLP formation was evident when purification was done in the absence of urea, except that yields were extremely low (data not shown).

\section{S antigen content of VLPs contributes to optimal EDIII accessibility}

Our objective was to display functionally competent DENV EDIII moieties on the VLP surface. Only then would such VLPs have potential utility for developing customized dengue-specific nanotools. To assess surface accessibility of EDIII, we employed a competitive ELISA wherein we measured the capacity of these VLPs to compete with free monomeric EDIII antigen for specific antigen-binding sites on anti-EDIII antibodies. In the experiment shown in Figure 5A, pre-incubation of the EDIII-specific mAb 24A12 with $\mathrm{S}$ antigen (blue curve) did not adversely affect its ELISA reactivity towards yeast-expressed DENV-2 EDIII-2 [26]. On the other hand, pre-incubation with the ES, $\mathrm{S}_{4}$ VLPs (red curve) produced a dose-dependent reduction in the capacity of the mAb towards recombinant EDIII-2, resulting in 50\% reduction at a concentration of $1.4 \mu \mathrm{g} / \mathrm{ml}$. The ELISA reactivity of this $\mathrm{mAb}$ upon pre-incubation with $\mathrm{ES}, \mathrm{S}_{0}$, $E S, S_{1}$ and $E S, S_{2}$ VLPs displayed profiles lying between these two extremes (data not shown). That all these VLPs did compete, albeit at different efficiencies, is clearly a reflection of the surface accessibility of the VLP-displayed EDIII. It is noteworthy that the relative proportion of the EDIII moiety is highest in ES, $\mathrm{S}_{0}$ and the lowest in ES, $\mathrm{S}_{4}$ VLPs. That ES, $\mathrm{S}_{4}$ despite its lowest EDIII moiety content manifests the highest efficiency in competing for $\mathrm{mAb}$ binding suggests that it provides for maximal ease of accessibility, and therefore, display of EDIII moieties, on the VLP surface. It automatically follows that, if EDIII is displayed on the VLP surface, it must be able to compete with infectious DENV-2 for host cell receptor-binding. To this end, we exposed Vero cell monolayers to the different VLP preparations followed by DENV-2 infection. Successful infection would be followed by viral replication within the cells. We followed this using viral NS1 antigen secretion in the culture supernatant as shown in Figure 5B. The data showed that the ES, $S_{2}$ and ES, $S_{4}$ VLPs were able to potently inhibit NS1 antigen secretion in contrast to the ES, $S_{0}$, and ES, $S_{1}$ VLPs, which were virtually ineffective in this regard. Once again, the inverse relationship between relative EDIII content and its functional competence of the VLPs was reflected in this experiment.

Taken together, the competitive ELISA and virus binding-blocking experiments support the notion that mosaic VLPs containing the ES and S antigens in 1:4 ratios display EDIII on the surface in a manner that is compatible with its optimal interaction with antibodies and the host cell receptors.

\section{Discussion}

The magnitude of dengue as a public health problem is accentuated by the lack of drugs and vaccines $[3,4,9]$. The emergence of nanotechnology and its increased

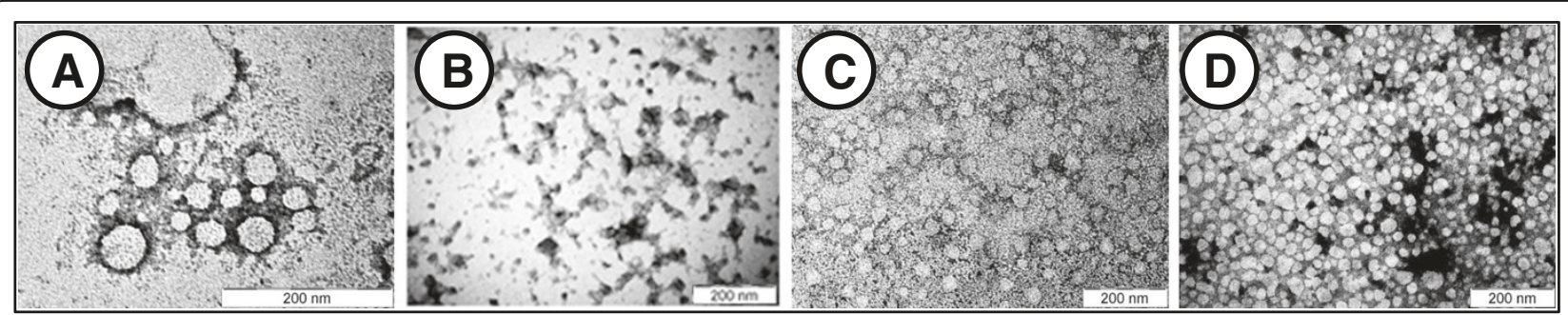

Figure 4 Electron microscopic visualization of purified ES,S antigens. The purified ES, $S_{0}$ (panel ' $\mathbf{A}$ '), ES, $S_{1}$ (panel ' $\mathbf{B}$ '), ES, $S_{2}$ (panel ' $\mathbf{C}^{\prime}$ ), and ES, $S_{4}$ (panel ' $\mathbf{D}$ '), antigens shown in Figure 3B were stained with uranyl acetate and visualized by electron microscopy. 

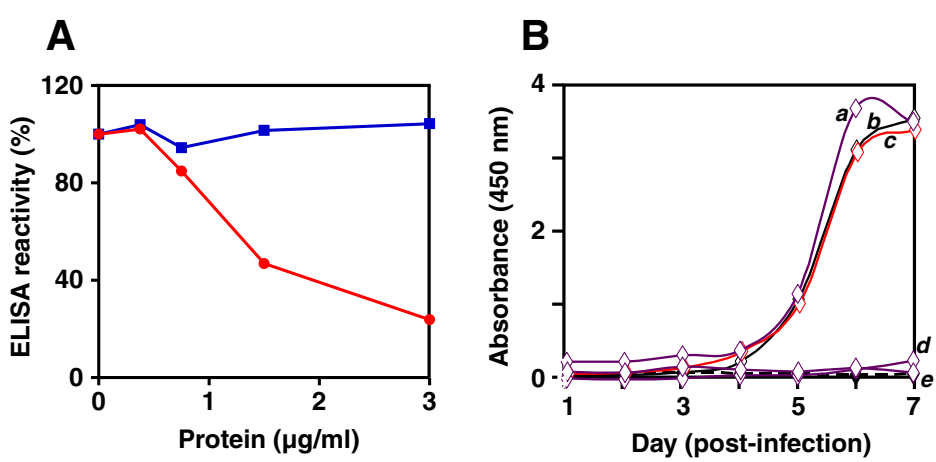

Figure 5 Functional characterization of the ES,S VLPs. (A) Competitive ELISA analysis. Anti-EDIII mAb was pre-incubated either with $\mathbf{S}$ (blue curve) or $E S, S_{4}$ (red curve) antigen VLPS, followed by titration of residual mAb reactivity on yeast-expressed EDIII-2 antigen-coated ELISA plates. (B) Binding blocking analysis. Vero cells were pre-incubated with S (curve ' $c$ '), ES, $S_{0}$ (curve ' $a$ '), ES, $S_{2}$ (curve ' $d$ ') and ES, $S_{4}$ (curve 'e') VLPs and then infected with DENV-2. Control DENV-2 infection in the absence of any VLP pre-incubation (curve 'b') and mock-infections (dashed curve) were carried out in parallel. The progress of infection was monitored over a week by measuring the release of NS1 antigen in the culture supernatant. Experiments in both panels $\mathbf{A}$ and $\mathbf{B}$ were repeated twice; results of one representative experiment each are shown.

recent focus on bionanomaterials has opened up new interdisciplinary avenues exploring novel biomedical applications [11-13]. Our interest is in exploring this area with the ultimate goal of developing nanotools that could help address the dengue problem. In this context, our interest is in the use of bionanomaterials which have the intrinsic advantages of biocompatibility and biodegradability [12]. In contrast to naturally occurring viral nanoparticles, which are biohazardous, their genomefree counterparts, the VLPs, are being increasingly preferred for developing biomedical nanotools [11-13].

The $\mathrm{S}$ antigen of Hepatitis $\mathrm{B}$ represents the classic example of a viral protein which can independently assemble into nanoparticles [19-21] and is the basis of a highly successful VLP vaccine [22,23]. We have sought to endow DENV specificity to these S VLPs, as a first step towards developing bionanoparticles with potential dengue-specific applications. To confer DENV specificity we chose a $\sim 100$ aa domain known as EDIII, for reasons mentioned already, and genetically linked it to the $\mathrm{N}$-terminus of the $\mathrm{S}$ antigen, and expressed it using $P$. pastoris. We observed that the resultant ES antigen did not form VLPs efficiently. Moreover, it did not display EDIII optimally based on specific assays to test its functionality. In order to obtain VLPs with functionally competent EDIII, we developed a strategy to co-express un-fused $\mathrm{S}$ antigen at calibrated levels using defined $S$ gene copy numbers. The resultant mosaic VLPs contain two components, ES antigen and un-fused $S$ antigen. Both ES and $S$ antigens tend to be membrane associated, by virtue of the latter's intrinsic hydrophobicity $[21,24,25]$. This necessitated their copurification starting from the membrane fraction using a protocol we developed recently for $\mathrm{S}$ antigen purification [21]. A noteworthy feature of this purification scheme is that it eliminates $\mathrm{CsCl}$ centrifugation, a bottleneck in downstream processing. Based on a variety of criteria including co-purification through $300 \mathrm{kDa}$ cut-off TFF, CsCl gradient sedimentation analysis, EM and sandwich ELISA's, we found that ES and S antigens associate together to form mosaic VLPs. Using competitive ELISA and bind blocking assays, we demonstrated that mosaic VLPs containing 4 copies of $S$ antigen per copy of the ES antigen displayed EDIII moiety optimally.

The data have significant implications from the perspective of dengue-specific nanoparticulate tools. As EDIII can induce virus-neutralizing antibodies [26,27], the repeat-pattern architecture of the mosaic VLP can potentially augment its vaccine potential. This notion is backed by the fact that vaccines based on the VLP platform are already available for Hepatitis B and human papilloma virus infections [13]. Strategies to load VLPs with drugs and target viral nanoparticles to tumors are being actively investigated [12]. Thus, if one can envisage charging these mosaic VLPs with a drug against dengue, these VLPs through their surface displayed EDIII can be targeted for drug delivery to DENV-susceptible cells through host receptor recognition. For vaccine and drug-delivery applications, the non-replicating, infectious viral genome-free VLPs offer the advantage of in-built safety. Additionally, as the EDIII is useful in serotype identification [27], these mosaic VLPs could also serve in diagnostic serotyping of DENV infections.

\section{Conclusions}

This work shows that DENV EDIII fused to the S antigen, co-expressed with un-fused $S$ antigen forms mosaic VLPs. VLPs containing the fused and un-fused components in 1:4 ratio displayed EDIII optimally. These EDIIIdisplaying VLPs have potential vaccine, drug-delivery 
and diagnostic applications. As these VLPs can be produced in P. pastoris, which is capable of high productivity in simple media, they can be expected to be inexpensive, a significant advantage for resource-poor regions where dengue is endemic. Finally, the approach developed here could serve as the basis for a common bionanoparticle platform for other infections as well.

\section{Methods}

The genes $E S(1 \mathrm{~kb})$ and $S(0.7 \mathrm{~kb})$, codon-optimized for P. pastoris expression were synthesized by Geneart AG (Regensburg, Germany). DENV-2 (NGC strain) stock was from previously reported work [28]. DENV-2 EDIIIspecific monoclonal antibody (mAb) 24A12 [26] and S antigen-specific mAb 5S [21] were in-house reagents.

A panel of four ES (1 copy) expression vectors, coexpressing $0,1,2$ and 4 copies of the $\mathrm{S}$ antigen was created using a head-to-tail in vitro multimerization method [19]. The ES gene was cloned into the unique Eco RI site of pAO815 to generate the expression plasmid pAO-ES, $S_{0}$. To provide for co-expression of $\mathrm{S}$ antigen, 1 , 2 and 4 copies of an $S$ gene expression cassette were inserted sequentially to generate pAO-ES, $\mathrm{S}_{1}, \mathrm{pAO}-\mathrm{ES}, \mathrm{S}_{2}$ and $\mathrm{pAO}-\mathrm{ES}, \mathrm{S}_{4}$, respectively. Each of the four constructs above was integrated into the genome of $P$. pastoris (GS115) as described before [19].

Typically, yeast cultures were grown at $30^{\circ} \mathrm{C}$ to $\log$ phase in buffered glycerol-containing medium (BMGY) and switched to buffered $1 \%$ methanol-containing medium (BMMY) for induction for 72 hours. Total (T) lysates for analytical experiments were prepared from methanolinduced cells using glass beads in a detergent-containing buffer as described [21]. A portion of the total lysate was separated by centrifugation into $\mathrm{S}$ - and the membraneenriched P-fractions. Suitable dilutions of T, S and P (ureasolubilized) fractions in urea-free buffer were used for sandwich ELISA and immunoblotting experiments.

For purification, we followed a method reported recently for $P$. pastoris-expressed $\mathrm{S}$ antigen purification starting from the membrane fraction [21] with some modifications as follows. Induced biomass (100 grams wet weight) was lysed with glass beads (5 cycles) in a Dyno-mill (WAB, Muttenz, Switzerland) and centrifuged to separate out the membrane-enriched $\mathrm{P}$ fraction, which was solubilized in a buffer containing $4 \mathrm{M}$ urea and $2 \%$ Tween 20 . The solubilized P fraction was clarified and subjected to 5\% PEG 6000 precipitation overnight. The post PEG supernatant was clarified by centrifugation and $0.45 \mu$ filtration and subjected to TFF across a $300 \mathrm{kDa}$ membrane using $12 \mathrm{~L}$ of TFF buffer with step-wise reduction in urea $(4 \mathrm{M}$ to $0 \mathrm{M})$. The TFF retentate was chromatographed on Phenyl $600 \mathrm{M}$ Toyopearl resin (Tosoh Bioscience, Stuttgart, Germany). Bound proteins were eluted using a 0-8 $\mathrm{M}$ urea step gradient (with $2 \mathrm{M}$ increase at each step lasting 5 bed volumes) in $20 \mathrm{mM}$ sodium bicarbonate buffer ( $\mathrm{pH}$ 9.6). Column fractions were analysed by SDS-PAGE, purified peak fractions pooled, and dialyzed against $1 \times$ PBS. In some instances, we also carried out purification from the $\mathrm{S}$ fraction in the absence of urea as described [25].

Proteins were characterized by ELISA, immunoblotting, $\mathrm{CsCl}$ gradient centrifugation and EM. ES protein was detected by sandwich ELISA using two formats. In one case, DENV-2 EDIII-specific mAb 24A12 was used for antigen capture, followed by revealing it with an $S$ antigen-specific mAb-enzyme conjugate. In the second case, both capture and reveal mAbs were $\mathrm{S}$ antigenspecific (from Hepanostika Ultra kit, Biomerieux, Marcy L'Etoile, France). Immunoblot analyses were performed essentially as reported earlier [16,21] using either $\mathrm{mAb}$ $24 \mathrm{~A} 12$ or $5 \mathrm{~S}$ mAb. Densitometric analysis of the resultant blots was performed using freely available image analysis software from $\mathrm{NIH}$ (ImageJ software). $\mathrm{CsCl}$ gradient fractionation was performed as described previously [19]. The presence of VLPs in the purified preparations was visualized by EM as before [16].

The functional status of EDIII moiety on the VLPs was assessed by its ability to (i) compete for binding to a specific $\mathrm{mAb}$ and (ii) inhibit DENV-2 binding to host cell surface receptors. Competitive ELISA was done essentially as reported [16] using mAb 24A12. Binding blocking assay was performed using Vero cells (American Type Culture Collection, Virginia, USA). Briefly, cells were pre-exposed separately to each of the VLP preparations for 1 hour followed by infection with DENV-2. Culture supernatants were sampled at daily intervals for release of viral NS1 antigen using Dengue NS1 Platelia kit (BioRad Inc., USA) as per the manufacturer's directions [29].

\section{Additional file}

Additional file 1: Word file containing more experimental details and supplementary data (Figures S1-S4).

\section{Abbreviations}

AOX1: Alcohol oxidase 1; DENV: Dengue virus; EDIII: Envelope domain III; ES: EDIII of DENV-2 fused to Hepatitis B virus surface antigen; ES, S $_{0-4}$ : ES antigen co-expressed with 0-4 copies of Hepatitis B virus surface antigen; PEG: Polyethylene glycol; S: Surface antigen of Hepatitis B virus; $S$

fraction: Supernatant fraction; $P$ fraction: Pellet fraction; TFF: Tangential flow filtration; VLP: Virus-like particle.

\section{Competing interests}

The authors declare that they have no competing interests.

\section{Authors' contributions}

Conceived and designed the study: SS and NaK; plasmid construction and integration into P. pastoris: NiK, AnP, SKN, ND, PN; expression optimization and purification: NiK, AnP, SKN, ArP, AsP and RV; EM analysis: PT and HL; Functional characterization: NiK, AnP, SKN, UA and RR; data interpretation: SKJ, UR, SS, NaK; wrote the manuscript: UR, SS and NaK; all authors read and approved the manuscript. 


\section{Acknowledgements}

$\mathrm{NiK}$ and $\mathrm{AnP}$ are recipients of junior research fellowships from the Council of Scientific \& Industrial Research, Government of India. SS and NaK are grateful to the Department of Biotechnology, Government of India for providing funds to perform this work. SS and NaK thank Drs. Harold Margolis, Dennis Trent, George Siber and Cristina Cassetti for their inputs in designing the mosaic VLPs.

\section{Author details}

${ }^{1}$ Recombinant Gene Products Group, International Centre for Genetic Engineering \& Biotechnology, Aruna Asaf Ali Marg, New Delhi 110067, India. ${ }^{2}$ Leibniz University of Hannover, Technical Chemistry-Life Science, Hannover, Germany. ${ }^{3}$ Helmholtz Centre for Infection Research, Braunschweig, Germany. ${ }^{4}$ Department of Biotechnology, Jamia Hamdard, Hamdard Nagar, New Delhi 110062, India.

Received: 7 March 2013 Accepted: 23 May 2013

Published: 25 May 2013

\section{References}

1. Gubler DJ: The economic burden of dengue. Am J Trop Med Hyg 2012, 86:743-744.

2. Gubler DJ, Kuno G, Markoff L: Flaviviruses. In Fields Virology. 5th edition. Edited by Knipe DM, Howley PM. Philadelphia: Wolters Kluwer and Lippincott Williams \& Wilkins; 2007:1153-1252.

3. WHO: Dengue and dengue haemorrhagic fever. 2012. Factsheet No117 2012 www.who.int/mediacentre/factsheets/fs117/en/.

4. Swaminathan $\mathrm{S}$, Khanna N: Dengue: recent advances in biology and current status of translational research. Current Mol Med 2009, 9:152-173.

5. Dussart P, Petit L, Labeau B, Bremand L, Leduc A, Moua D, Matheus S, Baril $L$ : Evaluation of two new commercial tests for the diagnosis of acute dengue virus infection using NS1 antigen detection in human serum. PLoS Negl Trop Dis 2008, 2:e280

6. Peeling RW, Artsob H, Pelegrino JL, Buchy P, Cardosa MJ, Devi S, Enria DA, Farrar J, Gubler DJ, Guzman MG, Halstead SB, Hunsperger E, Kliks S, Margolis HS, Nathanson CM, Nguyen VC, Rizzo N, Vázquez S, Yoksan S: Evaluation of diagnostic tests: dengue. Nat Rev Microbiol 2010, Suppl:S30-S37. doi:10.1038/nrmicro2459.

7. Noble CG, Chen YL, Dong H, Gu F, Lim SP, Schul W, Wang OY, Shi PY: Strategies for development of dengue virus inhibitors. Antiviral Res 2010, 85:450-462.

8. Pastorino B, Nougairède A, Wurtz N, Gould E, de Lamballerie X: Role of host cell factors in flavivirus infection: Implications for pathogenesis and development of antiviral drugs. Antiviral Res 2010, 87:281-294.

9. Swaminathan $\mathrm{S}$, Batra $\mathrm{G}$, Khanna N: Dengue vaccines: state of the art. Expert Opin Ther Patents 2010, 20:819-835.

10. Sabchareon A, Wallace D, Sirivichayakul C, Limkittikul K, Chanthavanich $P$, Suvannadabba S, Jiwariyavej V, Dulyachai W, Pengsaa K, Wartel TA, Moureau A, Saville M, Bouckenooghe A, Viviani S, Tornieporth NG, Lang J: Protective efficacy of the recombinant, live-attenuated, CYD tetravalent dengue vaccine in Thai schoolchildren: a randomised, controlled phase $2 b$ trial. Lancet 2012, 380:1559-1567.

11. Soto CM, Ratna BR: Virus hybrids as nanomaterials for biotechnology. Current Opin Biotechnol 2010, 21:426-438.

12. Yildiz I, Shukla S, Steinmetz NF: Applications of viral nanoparticles in medicine. Current Opin Biotechnol 2011, 22:901-908.

13. Chackerian B: Virus-like particles: flexible platforms for vaccine development. Expert Rev Vaccines 2007, 6:381-390.

14. Myyryläinen T, Talha SM, Swaminathan S, Vainionpää R, Soukka T, Khanna N, Pettersson K: Simultaneous detection of human immunodeficiency virus 1 and hepatitis B virus infections using a dual-label time-resolved fluorometric assay. J Nanobiotechnol 2010, 8:27.

15. Talha SM, Salminen T, Swaminathan S, Soukka T, Pettersson K, Khanna N: A highly sensitive and specific time resolved fluorometric bridge assay for antibodies to HIV-1 and -2. J Virol Methods 2011, 173:24-30.

16. Arora U, Tyagi P, Swaminathan S, Khanna N: Dengue virus type 2 envelope domain III displaying bio-nanoparticles. J Nanobiotechnol 2012, 10:30.

17. Sukupolvi-Petty S, Austin SK, Purtha WE, Oliphant T, Nybakken GE, Schlesinger JJ, Roehrig JT, Gromowski GD, Barrett AD, Fremont DH, Diamond MS: Type- and subcomplex-specific neutralizing antibodies against domain III of dengue virus type 2 envelope protein recognize adjacent epitopes. J Virol 2007, 81:12816-12826.

18. Crill WD, Roehrig RT: Monoclonal antibodies that bind to domain III of dengue virus $\mathrm{E}$ glycoprotein are the most efficient blockers of virus adsorption to Vero cells. J Virol 2001, 75:7769-7773.

19. Vassileva A, Chugh DA, Swaminathan S, Khanna N: Effect of copy number on the expression levels of Hepatitis B surface antigen in the methylotrophic yeast Pichia pastoris. Protein Exp Purif 2001, 21:71-80.

20. Gurramkonda C, Adnan A, Gäbel T, Lünsdorf H, Ross A, Nemani SK Swaminathan S, Khanna N, Rinas U: Simple high-cell density fed-batch technique for high-level recombinant protein production with Pichia pastoris: Application to intracellular production of Hepatitis B surface antigen. Microb Cell Factories 2009, 8:13.

21. Patil A, Khanna N: Novel membrane extraction procedure for the purification of hepatitis B surface antigen from Pichia pastoris. J Chromatog B 2012, 898:7-14.

22. McAleer WJ, Buynak EB, Maigetter RZ, Wampler DE, Miller WJ, Hilleman M: Human hepatitis B vaccine from recombinant yeast. Nature 1984, 307:178-180.

23. Shouval D: Hepatitis B vaccines. J Hepatol 2003, 39:S70-S76.

24. Biemans $R$, Thines $D$, Petre-Parent B, de Wilde M, Rutgers T, Cabeźon T: Immunoelectron microscopic detection of the Hepatitis B virus major surface protein in dilated perinuclear membranes of yeast cells. DNA Cell Biol 1992, 11:621-626.

25. Lünsdorf H, Gurramkonda C, Adnan A, Khanna N, Rinas U: Virus-like particle production with yeast: ultrastructural and immunocytochemical insights into Pichia pastoris producing high levels of hepatitis B surface antigen. Microb Cell Fact 2011, 10:48.

26. Batra G, Raut R, Dahiya S, Kamran N, Swaminathan S, Khanna N: Pichia pastoris-expressed dengue virus type 2 envelope domain III elicits virus-neutralizing antibodies. J Virol Methods 2010, 167:10-16.

27. Ludolfs D, Schilling S, Altenschmidt J, Schmitz H: Serological differentiation of infections with dengue virus serotypes 1 to 4 by using recombinant antigens. J Clin Microbiol 2002, 40:4317-4320.

28. Khanam S, Pilankatta R, Khanna N, Swaminathan S: An adenovirus type 5 (AdV5) vector encoding an envelope domain III-based tetravalent antigen elicits immune responses against all four dengue viruses in the presence of prior AdV5 immunity. Vaccine 2009, 27:6011-6021.

29. Korrapati AB, Swaminathan G, Singh A, Khanna N, Swaminathan S: Adenovirus delivered short hairpin RNA targeting a conserved site in the $5^{\prime}$ nontranslated region inhibits all four serotypes of dengue viruses. PLoS Negl Trop Dis 2012, 6:e1735.

doi:10.1186/1477-3155-11-15

Cite this article as: Khetarpal et al:: Dengue-specific subviral nanoparticles: design, creation and characterization. Journal of Nanobiotechnology 2013 11:15.

\section{Submit your next manuscript to BioMed Central and take full advantage of:}

- Convenient online submission

- Thorough peer review

- No space constraints or color figure charges

- Immediate publication on acceptance

- Inclusion in PubMed, CAS, Scopus and Google Scholar

- Research which is freely available for redistribution 